\title{
Design and Narrative Structure for the Virtual Human Scenarios
}

\author{
Stefan Göbel, Ido Aharon Iurgel, Markus Rössler, Frank Hülsken and Christian Eckes
}

\begin{abstract}
This article describes the design of the two application scenarios of the Virtual Human project and its integration into the Virtual Human system. This includes overall concepts and considerations of the demonstrators for the two application scenarios (learning, edutainment) as well as underlying methodic-didactic aspects for knowledge transmission and narrative concepts for story structure and story control during run-time of the system. Hence, in contrast to traditional learning systems with virtual characters as virtual instructors, an exciting and suspenseful interactive information space has been created. On the one hand, the methodic-didactic methods and VH learning model guarantee learning effects, on the other hand narrative structures and an emotion module provide the ground for a playful and exciting story environment, whereby the users can interact and discuss with a set of virtual characters.
\end{abstract}

Index Terms-Interactive storytelling, learning, narration, ontologies, virtual human.

\section{INTRODUCTION}

The methods and concepts provided in this article describe the backend of the Virtual Human system. Fig. 1 characterizes the workflow and interdependencies of the components provided by the authors of this article within the overall Virtual Human architecture:

With respect to the authoring process, $\mathrm{rmh}$ provides the overall application framework (VH design and set-up of the application scenarios) and Fraunhofer IMK elaborated a learning model and content specific ontology. This content is stored within the VH content base. ZGDV accesses this content via an authoring environment (VH editors) and puts everythin together to a story structure enhanced by narrative concepts. The storyboard as result of the authoring process builds the input for the Virtual Human run-time system. Here, the Narration Engine by ZGDV loads the story and controls the

Manuscript Received on March 25, 2007

Stefan Göbel received a diploma in computer science at the Technical University of Darmstadt in 1997. E-Mail: stefan.goebel@zgdv.de.

Ido Aharon Iurgel has a master degree in philosophy, social psychology. His special interests are concepts for the integration of humanistic sciences and computer science. E-Mail: iiurgel@zgdv.de.

Markus Rössler is one of the founders of rmh new media $\mathrm{GmbH}$. He is managing director and responsible for $3 \mathrm{D}$ content in the field of storytelling and virtual reality. E-Mail: roessler@rmh.de.

Frank Hülsken is a mathematician with background in pattern recognition. His research interests include speech recognition, image processing, 3D reconstruction and animation. E-Mail: Frank.Huelsken@imk.fraunhofer.de.

Christian Eckes received the diploma degree in Physics from the University of Dortmund in 1995. His research interests include human-computer interaction, multimedia analysis, pattern recognition and biological inspired computer vision. E-Mail: Christian.Eckes@imk.fraunhofer.de. system during run-time.

\section{DESIGN OF VIRTUAL HUMAN SCENARIOS}

During the Virtual Human research project, two demonstrator applications have been developed to test the technologies, tools and paradigms of the project. Apart from mere technological validation, the demonstrators served to investigate the market potential and end-user acceptance of agent-based, multi-modal applications.

The established practice of validating the technology developed during a project by building a single demo application at the end of the project was broken down by the Virtual Human team. Contrary, the focus was set on integration from the beginning of the project and a first early demonstrator has been established after a one-year development phase only. This was possible due to the available technology by the different project partners of the Virtual Human consortium (e.g. the rendering system AVALON provided by Fraunhofer IGD, dialogue-based story engine from ZGDV or the CharActor engine by Charamel).

Based on that early demonstrator the integrated VH system has been enhanced by new methods and concepts and a second demonstrator has been established, also serving as basis for market studies and the project evaluation process. In the following sections the Virtual Human demo applications are described in detail from the viewpoint of a concept and content creator, focusing on design considerations and story logics.

\subsection{General Scenario}

The challenge consisted in combining the concepts of multi-agent systems represented by virtual avatars and multi-modal user interaction. The general conceptual framework was similar for both applications: One or more players would interact with two virtual characters, one playing a dominant role (teacher or presenter) and one on par with the player (pupil or contestant). The virtual characters would interact with each other as well as with players through speech, gesture and facial expression. They would have no deeper knowledge about each other apart from the information they could extract from interaction and react emotionally to their counterparts (virtual and real). This simple setup offered the opportunity to investigate a number of interesting relationships. In most projects including avatars, a player is either faced by a single 'expert' character that is mainly a representation of an information system that a user can query or be taught by, or by a larger number of avatars that are just virtual representations of other real players (mostly used in multiplayer simulation, strategy and online role-playing games such as Sims, World of Warcraft or Second Life). The Virtual Human applications 


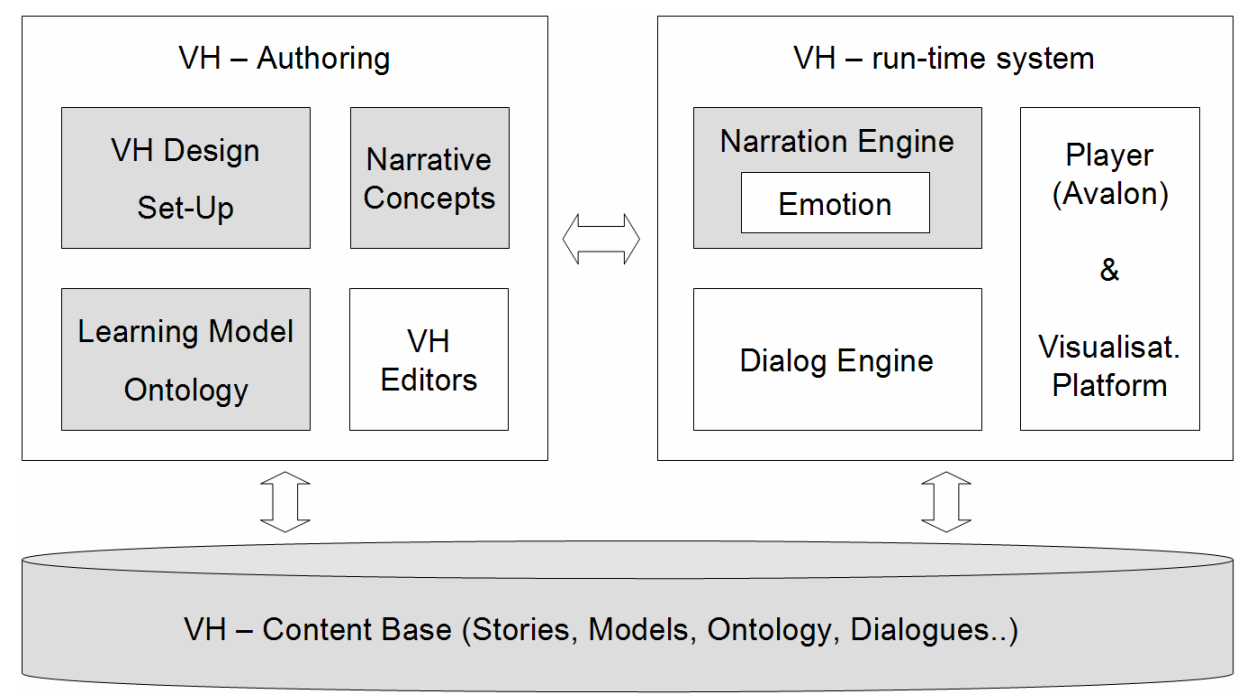

Fig. 1. Backend of the Virtual Human system - integration of application design, methodic-didactic and narrative concepts.

added a second virtual character to the interaction thereby creating a triangle of tension as not all characters were equal. This added a new level of believability to the virtual characters by stirring emotions in the player that are untypical for computer applications (e.g. compassion, anger, jealousy). Players interacted with the virtual world either by using natural language or via more traditional input devices such as a trackball, depending on the setup.

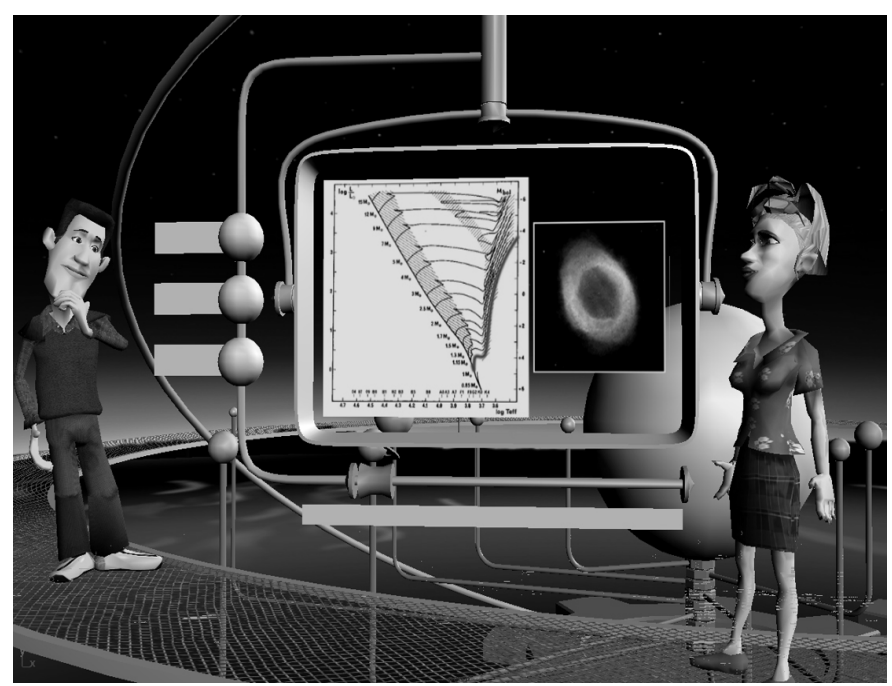

Fig. 2. Screenshot from the 'Life of a Star' demonstrator.

\subsection{Scenario Requirements}

Each demonstrator should have a very focused scenario in order to keep the complexity of the story manageable and it should be presented as a self-contained world. This made it easier to create a visually appealing set and cast, considering the limited resources of a demo application. One 'playing' session should last around 15 minutes in order to allow a reasonable throughput at public presentations and during evaluation sessions. The interaction paradigms and techniques should be intuitive and exhibit a steep learning curve to guarantee user satisfaction considering the short duration of a session. Finally the demonstrators were considered a means of exploring business opportunities for agent-based multi-modal systems. Therefore the scenarios should be selected from commercially promising areas and be easily adaptable to varying setup conditions (e.g. fixed museum installation, short-time trade-fair setup etc).

\section{3 'Life of a Star' Scenario}

E-learning was identified as a promising application field for multi-modal interaction. Most classic e-learning applications featuring avatars are based on the principle of a single teacherpupil relationship ("Frontalunterricht"). Pedagogical research has shown that this approach is far from ideal (see Section III). Adding a third character in the form of a fellow pupil offers players the opportunity to compete against someone and to create an emotionally charged situation.

The subject of the virtual science class was the 'life of a star' (see Fig. 2). It was chosen because it can be treated on a number of different levels and is clearly delineated. On the simplest level, the story was a linear progression from the birth of a star to its death, illustrating the most important stages with images, HR diagrams and spoken explanations. At predetermined points the storyline was interrupted by multiple-choice questions that were either directed at the real pupil or their virtual counterpart. Performance in these mini-tests influenced the depth of information presented by triggering respectively skipping story loops covering more advanced topics. One class session consisted of a complete lifecycle, its duration depending on the skill level of the player.

The user communicated with the teacher through natural language recognition, allowing them to freely formulate their answer without artificial restrictions. The teacher character reacted emotionally to the pupils, praising them for correct answers and rebuking them for wrong ones. This emotional information was processed by the pupil and controlled its gestures and facial expressions. 


\section{4 'ZAMB' Scenario}

While the main focus of the early demonstrator lay on technical issues, the final demonstrator aimed at creating a compelling user experience and reference application that could be presented to potential customers. Preliminary evaluation of the early demonstrator made clear that the theme of the application would need to be even tighter; that informal communications should be part of the scenario and that one has to choose even more emotionally laden subject that science.

Among a number of different proposals, football was chosen as the ideal candidate, not only because of the FIFA World Cup 2006 in Germany during the Virtual Human project period, but also due to the fact that football is a "hot and emotional topic" engaging many people. Especially when it comes to matches of the national team, almost everyone has to say something and more or less take the role of the team coach. Subsequently, the name of the second application scenario for the Virtual Human approach has been titled "ZAMB - Zweiundachtzig Millionen Bundestrainger" (engl. 82 million coaches). Apart from the research oriented facilities, emotional topics such as soccer or sports in general are also very interesting with respect to commercial aspects: Broadcasting rights, advertisement and branding or merchandising.

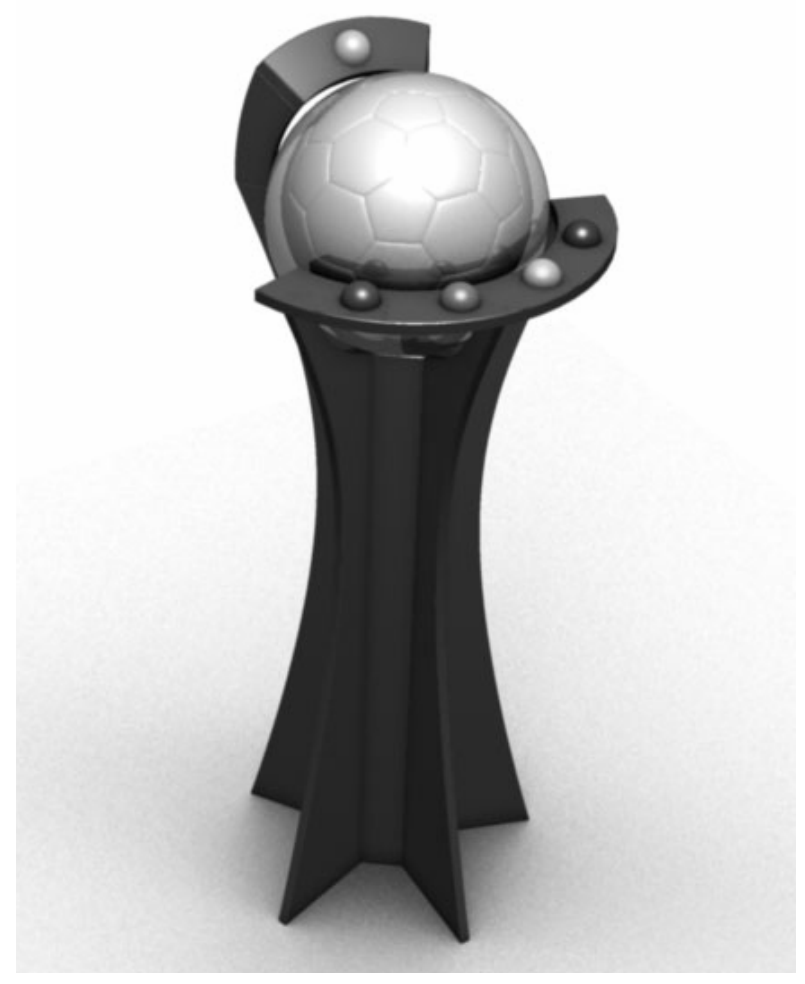

Fig. 3. Design study for the ZAMB trackball controller.

ZAMB was conceived as a modular quiz show on soccer topics that would take place in a virtual tv sports studio. It was made up of two parts that could be played in sequence or as individual games, allowing the game complexity and session length to be adjusted. The virtual cast consisted of a host, a football expert and a guest. Interaction took place between all of the virtual characters and one or two real players. Similar to a real talk-show host, the host character guided the players through the game, initiating actions, getting people to participate and commenting on their performance. Two interfaces between the real and the virtual world were developed. The first was based completely on speech using natural language recognition for player input and speech synthesis for output (combined with gestures and facial expressions). Unfortunately there are still many situations where voice recognition does not work robustly enough (e.g. trade-fairs). Therefore an alternative input device was constructed in the form of a large trackball with buzzers, featuring a design reminiscent of a football trophy (see Fig. 3).

In the first game, players were shown an extract from an international soccer match and were then asked to guess how the scene would develop, given three choices. Two real players and the two virtual guests participated in the game, commenting on each other's performance.

The (real world) winner of the first game was invited to the second round, where they had to setup the german team for an important international game. As they assigned players to positions, their choices were rated in real-time and commented upon by the host. Ratings depended on the overall tactical setup (e.g. 4-3-3), individual appropriateness for a given position and player combination strengths.

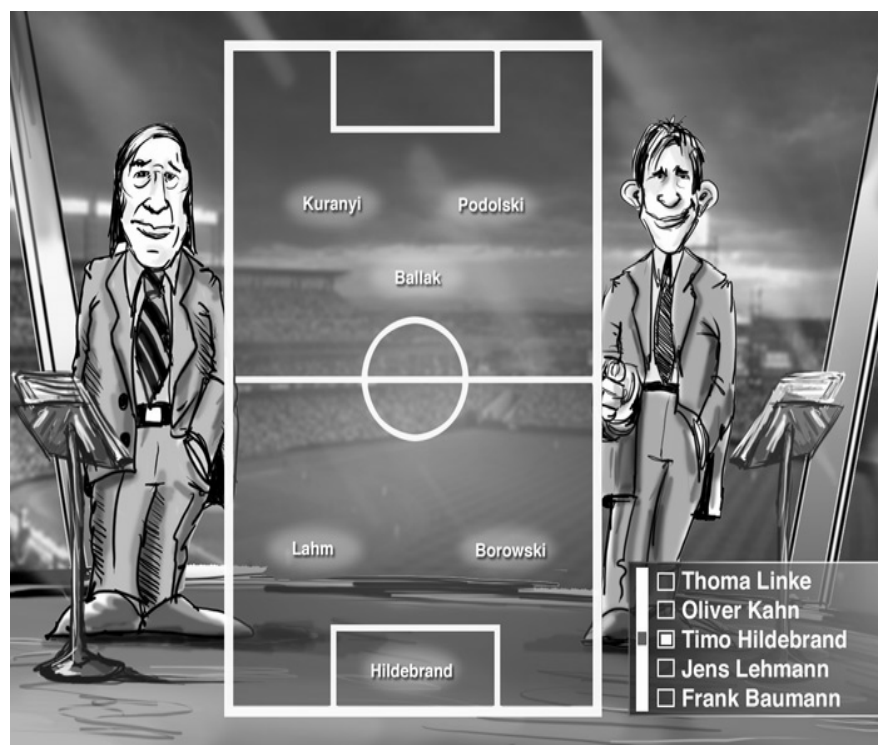

Fig. 4. Concept drawing for the ZAMB demonstrator.

\subsection{Virtual Set Design}

At the beginning of the project -due to the fact that were was no predefined storyboard with scripted dialogues etc.-different general considerations about the design of the virtual set has been arising: For instance, the avatars had to be positioned such that they could easily communicate with each other without turning their back onto each other or the audience. Characters needed to have enough room for gesturing without hitting anything. Virtual projection areas had to be integrated into the set design such that they would be visible from each character position fit the overall design and be adequately large for the (real) audience to distinguish critical features. In general the 
longtime experience of rmh of working with virtual studio sets proved very hand as requirements and constraints are very similar.

The set for the early demonstrator took up the subject of astronomy. As the vastness (and emptiness) of outer space is hard to capture in a static set, a vintage model of the planetary system was chosen as the structuring element of the studio. The brass tubes of the large-scale mechanical Kepler model created a strong contrast to the dark background and added a manageable level of depth to the scene that was especially important as the application was to presented as a stereoscopic projection. Furthermore it offered players a familiar element that they could (metaphorically) hold on to during their journey through the depths of astrophysics. Finally the virtual projection screen fit perfectly into the metallic mechanical setting of the Kepler model and could be rotated off-screen when not needed.

The ZAMB set was designed along the same lines as a typical virtual sports studio. It offered two connected areas, one for each game, laid out to allow the camera to pan from one to the other. The ZAMB logo was placed prominently in the scene to strengthen brand recognition. As three characters had to share the limited virtual studio space, control monitors were placed in the foreground to keep characters facing the audience when a movie was projected onto the background screen. The second game featured a large panoramic view into a (3d) soccer stadium paired with subdued ambient crowd noise. This well-known setting immediately established the scene as taking place shortly before the game begins when the official team structure is revealed.

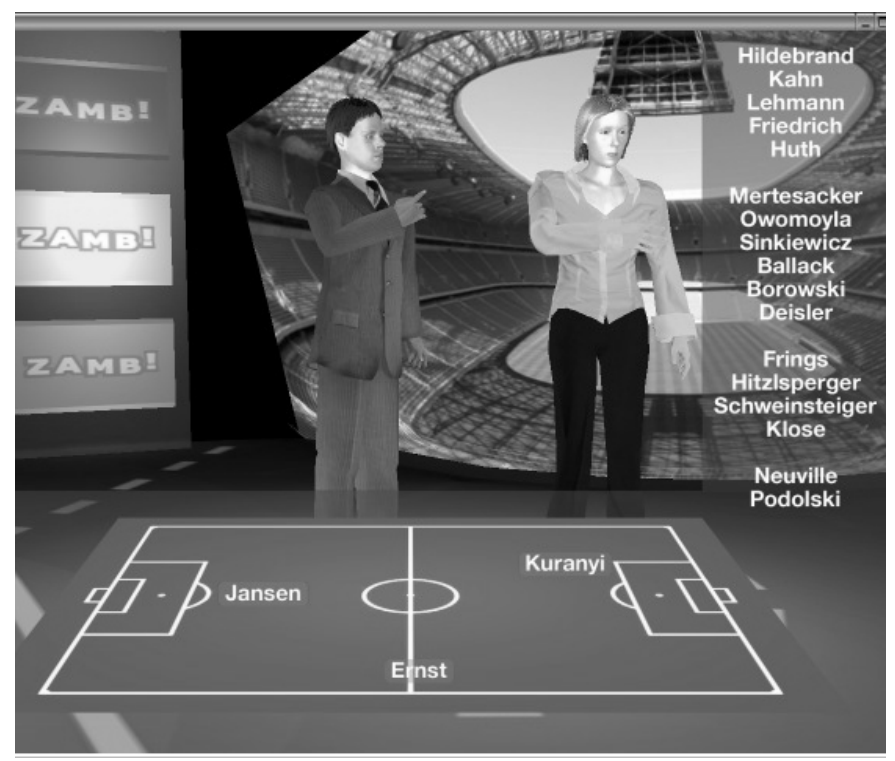

Fig. 5. Snapshot from the ZAMB demonstrator.

\subsection{Storyboard}

Once the general concept of an interactive installation has been developed, it is elaborated in the form of a storyboard. In the case of the Virtual Human demonstrators, a hand-drawn storyboard was created to establish the virtual sets the story was going to unfold in and to plan the high-level time structure.
Although the basic structure of both demonstrators was linear, the completely non-linear interaction between characters could not be easily captured by a traditional storyboard as complexity grows exponentially with the number of possible actions and reactions. A more object-oriented approach would be needed to allow enough depth to create believable characters and situations. Therefore a first version of the ontology used to drive the narration engine was developed by $\mathrm{rmh}$, based on the template developed during the SMARTKOM project. This draft represented the story world from the point of view of content creation and was restructured and extended in the next step to fit the technical needs of the narration engine.

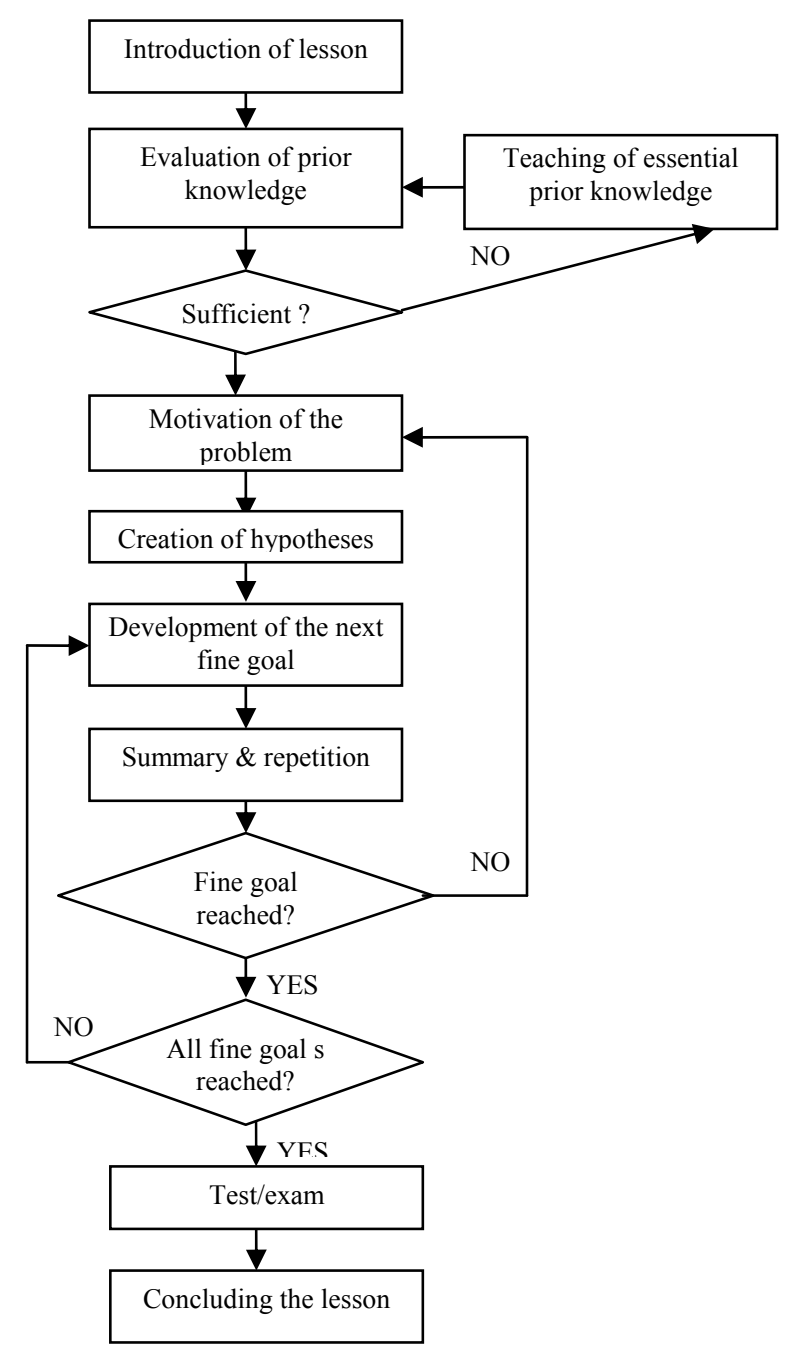

Fig. 6. Pedagogical model.

\section{PEDAGOGIC MODEL AND ONTOLOGY}

Before virtual characters can act as teachers in education, they have to be equipped with knowledge what and how they ought to teach. We have shown that knowledge about the topic can be provided in an appropriate format by developing an ontology, in which concepts and relations encode the topics the teacher wants to lecture about. However, this is not sufficient, since the representation still lacks information on how the virtual character should perform the teaching. Obviously, the 
system lacks general knowledge on the process of teaching and learning in general. We have to add information describing how such lessons is organized and what pedagogical strategies should be followed in our VR application to simulate a successful lesson. In the following we will report on how such models are designed in general, how the emotional state of the participants can be incorporates and how this can be formulated in terms of ontology. This work has also been published as [3], more general ideas can be found in [10].

\subsection{Pedagogical and Didactic Models}

There are quite different approaches of didactical models in educational applications, e.g. in educational games [1], classroom teaching [2] or for mission rehearsal [5]. The simplest strategy is characterized by the terms "Drill and Practise", often used in vocabulary training programs. As the title already indicates, it is based on a sequence of question and answering (QA) patterns during a lesson. It is obvious, that only simple declarative knowledge, such as multiplication tables, the names and dates of historic places, vocabulary and other "fact knowledge" can be taught that way. Moreover, such a method does only work for highly motivated pupils.

More recent, up-to-date methods for learning and teaching are based on the paradigm of active problem solving in which the problem is solved by formulating and refining working hypothesis in collaboration with the teacher. The quality and soundness of the resulting explanations are investigated by verbal or real experiments aimed to discard or support the suggested solutions. We chose to follow this idea by designed a didactical model for the virtual planetary in which the solar system and the development of stars are explained to the pupils. More background on didactical methods in physics can be found in [8] and [9].

\subsection{The pedagogic model of a lesson}

The lesson starts with an introduction of the topic. See also Fig. 1. The first step is to check whether the pupil has enough background knowledge to understand the upcoming subject. If this is not the case, the missing facts are taught. The lesson continues by letting the teacher motivate the current subject in the curriculum, e.g. by presenting the underlying problem in form of open questions. Teacher and pupil may jointly generate hypotheses for solving the problem. The solution of a problem represents a so called "fine goal". After reaching the fine goal, the teacher gives a short summary of the solution to deepen the pupils' understanding of the subject. This is followed by testing how much of the topic the student has understood and memorized ("goal controlling"). If the student fails this test, the steps of fine goal learning must be repeated. This cycle of defining fine goals and reaching them is repeated until all sub goals of the current lesson have been achieved. The lesson is concluded with a final test by which is pupil is evaluated and comes to an end by closing words of the teacher.

\subsection{Emotional representation of pupil and teacher}

Emotions during the school lesson emerge in teacher and virtual student and play an important role in simulating a realistic interaction. As this topic will also be discussed in more detail in other articles within this publication, let us only motivate what kind of actions may trigger what kind of emotions (see TABLE 1):

TABLE 1: CORRESPONDENCE OF EMOTION TO ACTION

\begin{tabular}{|l|l|l|}
\hline Action & Type of emotion & Intensity \\
\hline Teacher asks question & hope & 7 \\
\hline New topic & Joy & 4 \\
\hline Student gives right answer & Satisfaction & 5 \\
\hline Student gives wrong answer & Disappointment & 5 \\
\hline Student ignores teacher & Anger & 7 \\
\hline $\begin{array}{l}\text { Student talks with other } \\
\text { students }\end{array}$ & Anger & 6 \\
\hline Student runs around in class & Anger & 6 \\
\hline $\begin{array}{l}\text { Student gives useless } \\
\text { comments }\end{array}$ & Anger & 5 \\
\hline
\end{tabular}

The appraisal-model of Ortony, Clore and Collins (OCC) [12] is able to model the generation of such emotions. Such an appraisal model has to be combined with the pedagogical model to generate an appropriate action of the virtual characters in these situations. However, it is obvious that a reasonable pedagogical model prevents the teacher from giving away too much emotion.

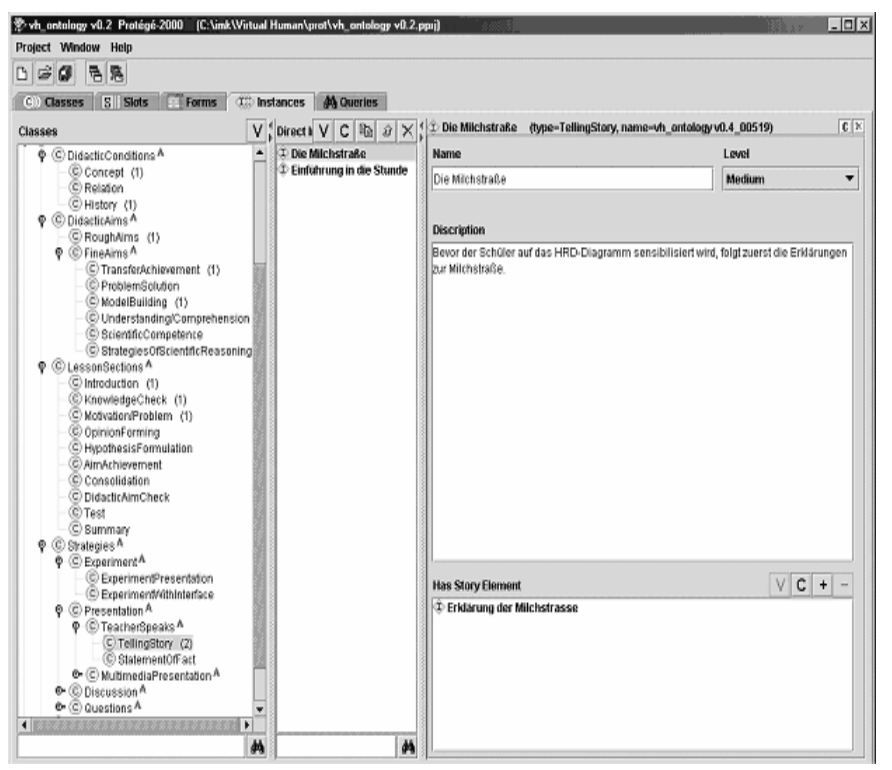

Fig. 7. Pedagogical model as an VH-ontology in Protege.

\subsection{An ontology for a lesson on astrophysics}

The main ontology consists of four different components. Classes for the representation of the lesson, described in 2.3, $3 \mathrm{D}$ world elements, like slider and textures, astrophysic objects and classes from the storyboard as an initial explanation. All components have links to the other classes, so that it is possible to bind a lesson element, which is part of a global narration concept, with a 3D model, which is an astrophysics object. To build an education model inside ontology you need five main classes:

- Lesson sections

- Didactic conditions

- Didactic aims 
- Order of strategies

- Strategies

Before a lesson could start the teacher has to fill these classes with his developed concepts. But he has also filled in additional ways of teaching, which could be choosing, if the student has problems. To finally arrange the seminar the approaches have to be weighted.

\subsubsection{The lesson section}

In this group we find the theoretical concepts described above.

TABLE 2: THE BASIC CLASSES FOR A LESSON

\begin{tabular}{|l|l|}
\hline 1) Introduction & 2) Knowledge Control \\
\hline 3) Motivation / problem & 4) Opinion forming \\
\hline 5) Hypothesis forming & 6) Development of aims \\
\hline 7) Consolidation & 8) Didactic aim control \\
\hline 9) Test & 10) Summary \\
\hline
\end{tabular}

In TABLE 2, we list the basic classes for a lesson, which could be filled with different strategies for one lecture.

\subsubsection{Didactic Conditions}

Didactic conditions are about the qualification the student has to have, before the teacher could go on with new learning matter. Here we have three different characteristics: Concept, Relation and History. A concept condition includes conception questions, a relation condition ask for the connections between other topics and a history condition ask for a placement in term of the background.

\subsubsection{Didaction aims}

To structure the lessons the teacher has to define the aims, he wants to achieve. We have rough aims, which are global concepts and fine aims. The fine aims are divided into Transfer attainment, problem solution, model forming, understanding/ cognition, science competence and strategies of science.

\subsubsection{Order of Strategies}

There are two orders of the strategy, the primary and the secondary one. If the primary strategy fails a secondary strategy could be tried. If both fail the next best strategy have to be calculated by the system taking into account the guidelines of the teacher and the preferences of the student.

\subsubsection{Strategies}

To achieve the aims of the lesson the system has different kinds of strategies, which the teacher arranges inside the ontology. Which strategy is chosen depends on several criterions: First of all the teacher configures his guidelines how a lesson should look like. Beside this he identifies bypasses. The student has an user profile, in which his preferences and also the strategies he responds best are stored. Furthermore the time left and the narration concepts could affect the choice. The strategies are divided into four sections:

- Experiment

- Presentation

- Discussion

\section{- Questions}

An experiment could just be a presentation or the student could active joined the experiment by using an interface. A presentation is performed by the teacher in telling a story or making an assertion. Or it could be a multimedia presentation and the teacher shows something on video, audio, at a 3D model or on a blackboard. A discussion could be controlled by the teacher or he lets the students discuss among each other. Strategies with questions are mainly used. Beside the simple question and answer pattern we have a multiple choice test and a task sheet the student has to fill out. These methods are used to get feedback, if the aims are successfully fulfilled to go on further.

\section{NARRATION}

Within Virtual Human the functionality of narration is two-fold: It concerns both story structuring in the authoring phase and story control during run-time.

The simple task is to combine the overall description and requirements of the application scenario and design of the virtual set with the learning model and the methodic-didactic aspects (what and how to transmit content) and make an exciting and suspenseful story out of it. Further the story should not be linear, but non-linear and interactive with a high degree of user involvement.

Based on that situation a narrative structure has been elaborated, whereby the structure primarily follows the learning model (in the case of the 'Life of a Star' scenario of the $\mathrm{VH}$ early demonstrator) respectively the game-level oriented ZAMB scenario. Fig. 8 shows that structure with an introductionary scene explaining the user the scenario and handling of the system, the first game and the second game as well as a 'Good Bye' scene at the end of the game-oriented ZAMB scenario.

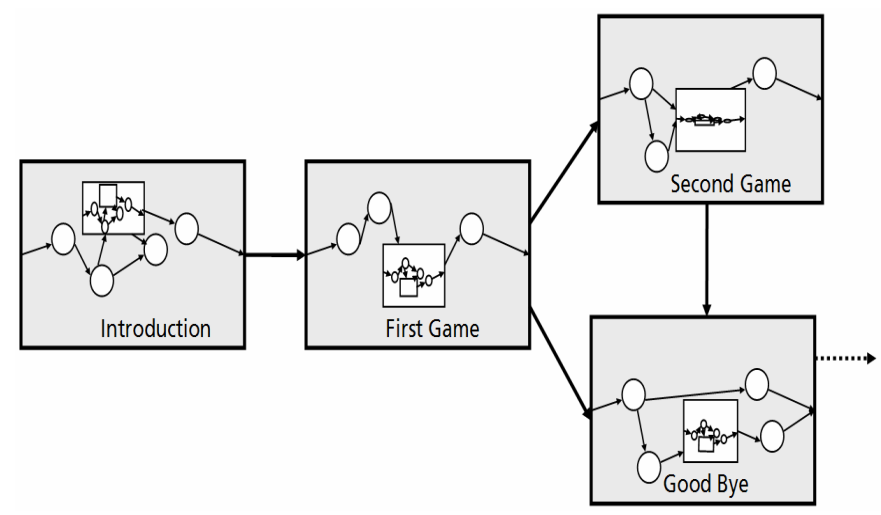

Fig. 8. Narrative structure of the ZAMB scenario.

Within the scenes, dialogue and gameplay takes place considering pre-defined content (sentences, dialogues among virtual characters) and user interactions (questions, directions, point-and-click; in summary any kind of multimodal input).

Hence, users can influence the flow and appearance of individual scenes (game levels), whereas the overall structure with the different scenes and transitions inbetween is 
predefined. However, the user has the chance to skip the second game and directly come to the end of the story.

\subsection{Authoring narrative structures}

With respect to the creation of such interactive application scenarios like ZAMB the main challenge concerns authoring tools and 'appropriate' mechanism to support authors, especially creative knowledge workers with no programming skills.

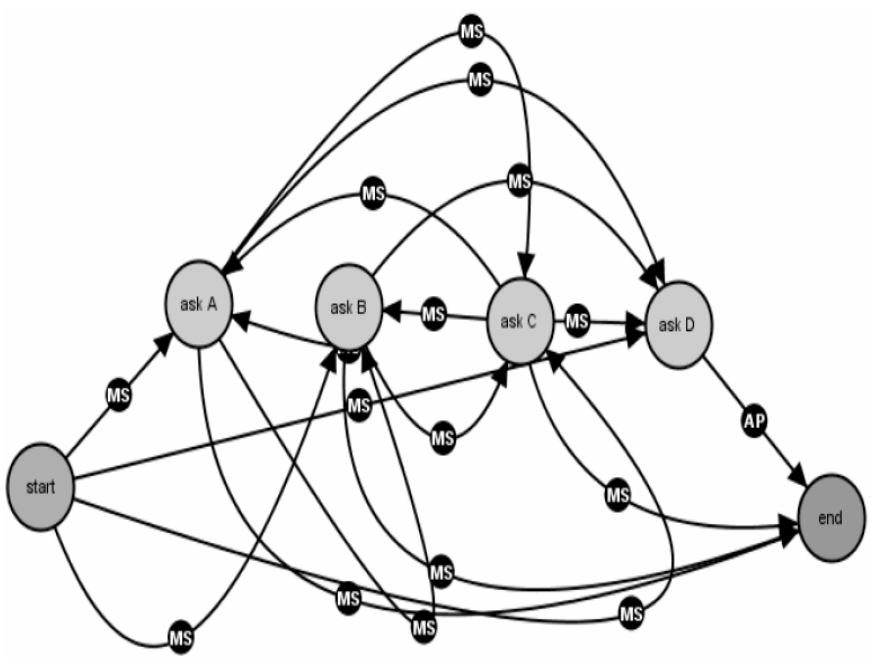

Fig. 9. Example of a dense story graph.

For this, recently a lot of research effort has been investigated to the development of authoring tools for interactive storytelling. Mateas' and Stern's work represent important advance in Interactive Storytelling, but the creation of interactive stories still is an enormously complex endeavor and requires a lot of programming [11]. No commercial authoring tool offers a satisfactory solution for the problem of enabling easy but powerful visual authoring. For instance, the standard tool for multimedia applications, Macromedia Director, offers a time line and enables to employ scripting; but scripting representss a switch out of the visual support into pure programming.

In order to provide visual support in the authoring process, graph-based approaches are most popular. Hereby, directed graphs are appropriate and sufficient in many contexts, but the disadvantage is that they easily become very dense and the guard conditions for the transitions very complex. Especially in highly interactive systems such as Virtual Human, directed graphs only have a limited use and further concepts are needed.

For that, since a couple of years ZGDV has been elaborated an authoring environment called Cyranus in strategic research and used it in the Virtual Human project apart from pure graph-oriented Virtual Human editors (see Fig. 10).

Cyranus offers a hierarchic transition network, which is derived from Harel's State Charts [4]. Additionally, Cyranus allows the activation of a state without using a transition. The main activation engine is the Jess engine that employs the rule system Jess (cf. Herzberg.ca.sandia.gov/jess). Iurgel provides further detailed information about Cyranus and its usage within interactive educational installations [6,7].

In order to retain the encapsulation of the composite states, and in order to allow for the concurrent use of different activation engines, an activation engeine is attached to exactly one specific composite state, and the states that can be activated are exactly the direct daughter states of this composite state.

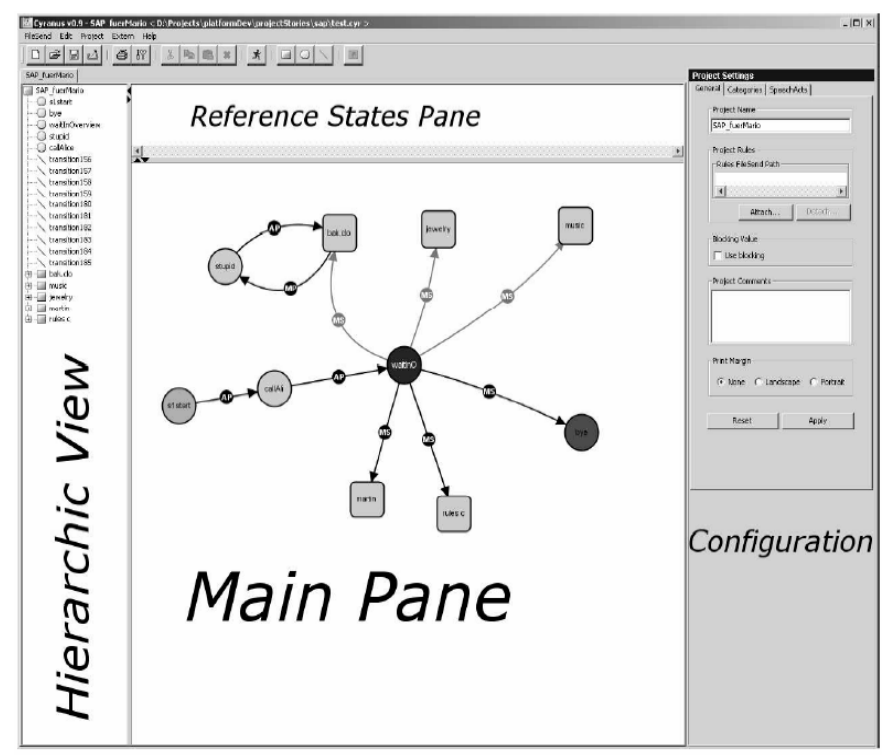

Fig. 10. Snapshot of the Cyranus authoring environment.

Each activation engine has its own data storage, keeps a representation of the meta data of the states, and is informed about every external event of the system. Several activation engines can compose a hierarchic chain, following the hierarchy of the composite states. These engines are called down-to-top, when no guard condition of a transition is passable, until some engine can handle the situation (or the system gives up). Internally, the guard conditions are kept as extended expressions encoded in XEXPR (www.w3.org/TR/xexpr) as a very powerful, XML based functional programming language.

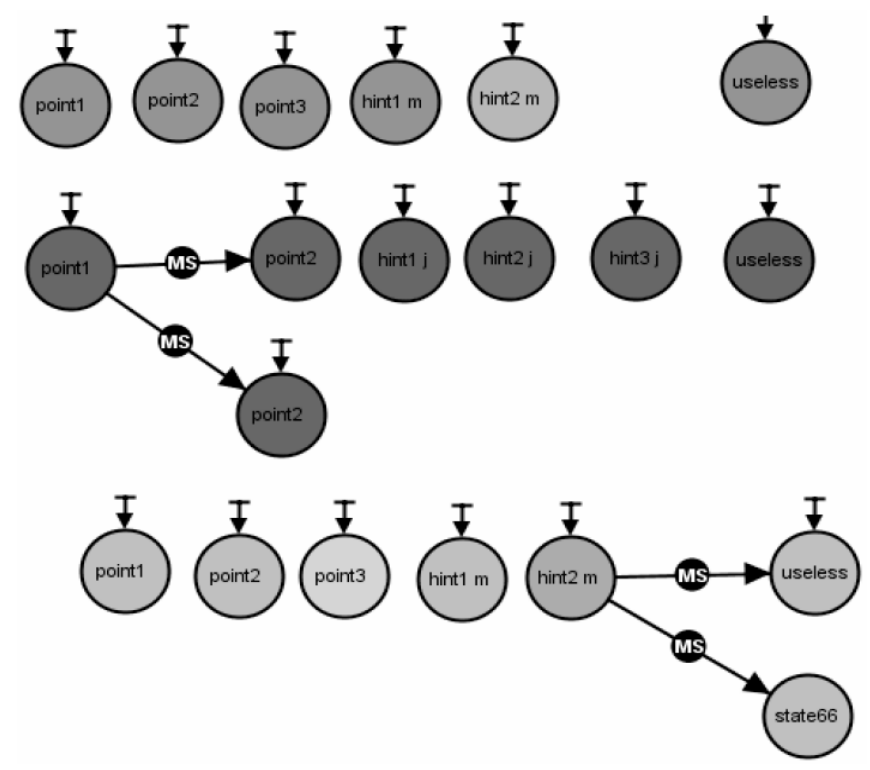

Fig. 11. Screenshot of Cyranus - employing an activation engine. 
Fig. 11 shows the visual representation of terminal states, which are not attached to transitions and being tagged by an arrow on top. This arrow symbolizes that the terminal states contain metadata, and subsequently can be activated by an activation engine.

In sum, there is an obvious analogy between the authoring concepts of Cyranus (hierarchic transition network plus activation engines: rule based system, etc.) and the logic of common learning adventure games. Similar, a top level composite state in the hierarchic transition network can be regarded as a 'scene' of a dramaturgy, or as a 'lesson' of a learning application. Hence, if the topology of states and transitions, and the guard conditions become too complex, it is possible to employ instead or additionally story and learning models, recurring to an activation engine, finally to control the sequencing.

\subsection{Narration Engine}

In Virtual Human, ZGDV developed a narration engine consisting of three levels: A hierarchic transition network on top level, a Jess based rule system at second level and a third level for Case-Based Reasoning (CBR).

As described in the authoring part in the previous section, the working principle is simple and follows a delegation chain: An external event that does not lead to the activation of a state within the transition network framework is passed over sequentially to other activation engines, which in their turn could activate a state, in this case breaking the chain of delegation. The engines are also permitted to fire without the occurence of an event. Every activation engine is attachached to a single composite state, through metadata of this state, and it my activate only terminal or composite states that are located within this composite state. Thus, encapsulation is fully preserved.

For the ZAMB scenario, two activation engines are employed: The production system Jess and a similarity based engine that implements CBR principles. Additionally, as a fall back engine (for the case that none of the three levels with the HTN and the activation engines match a transition to a well-defined next state), the chatterbot Alice (www.alicebot.org) has been employed. Alice is not an activation engine, because it does not activate a state that is within reach of all activation engines. Instead, the Alice Engine generates autonomously an action that is sent to the client.

\subsection{Emotion Module}

One of the major issues of the Virtual Human approach targeted the realistic presentation of virtual characters, not only concerning the graphical part with real-time rendering etc., but also referring to realistic conversation forms taking into account mimics, gestics and emotions.

In daily life, interacting with a person that is fulfilling a certain duty-a functionary or employee, a sales person, a teacher, a work colleague-normally involves more than purely accomplishing rationally "hard" transactions. "Soft", emotional factors play an eminent role for the success and the stability of the processes. For instance, a teacher should expect better learning results and continuity of work if the students feel at easy with him or her; a sales person is more successful if the customer feels sympathy and laughs at the jokes of the vendor. In short, soft, social and emotional skills matter very much, in daily life.

Their importance for the efficient operating of organizations and for the accomplishment of rational tasks in a social context is well known and studied. Vice versa, this is not the case for virtual characters in virtual environments. Currently available embodied virtual assistants show only very rudimentary soft skills and personality traits. For example, a virtual shopping assistant usually displays a constant smile as the only sign of emotional involvement, remaining otherwise passive and emotionally shallow.

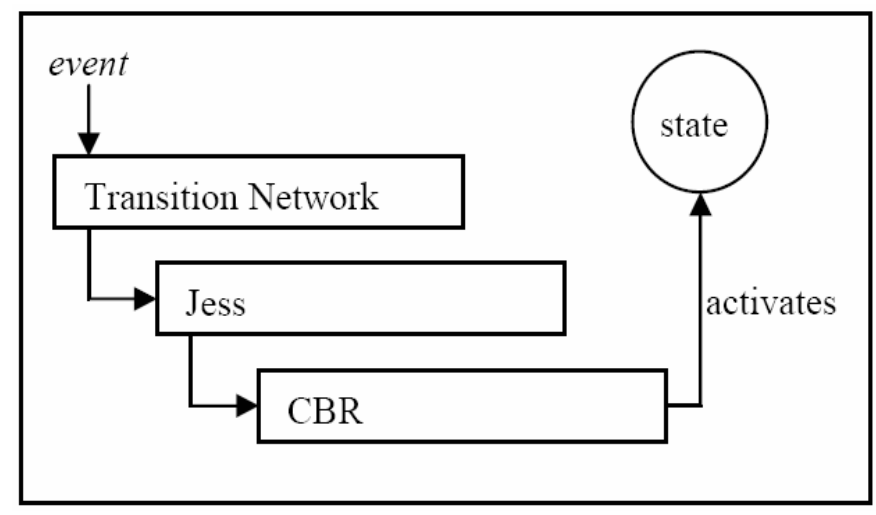

Fig. 12. Narration Engine with a three level delegation chain.

The question is what exactly are the soft skills required for a technically feasible virtual character? Which kind of emotional, not task oriented behaviour is useful, and what is superfluous or even disadvantageous?

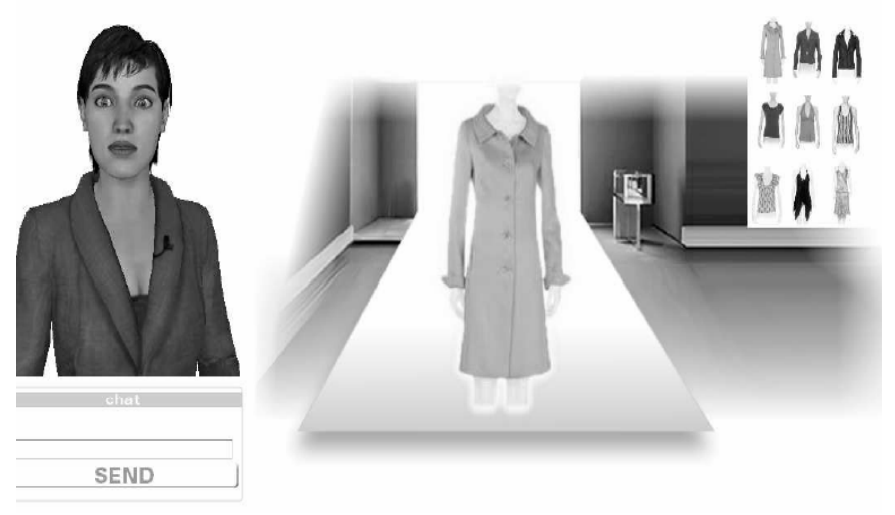

Fig. 13. Virtual Human with Social Intelligence - Julie's Store.

For the ZAMB scenario in Virtual Human, the characteristics of the virtual characters have been built on observations from real TV shows about soccer, especially analysing the "soft" and emotional parts of the different roles: Moderator, expert, guest. Some of these characteristics have been rudimentary transmitted into rules, which are considered by an emotion module as part of the narration engine. For instance, the moderator brings in a joke, when the expert and guest are "too boring" or the situation becomes too delicious in the sense of a conflict. Hereby, the basic problem from a 
research driven perspective is to evaluate the effects of emotional virtual characters to users-which effect does a joke have to an individual user? This is very difficult to foresee and the analogy between a virtual and a real human can be misleading. If the user laughs at a joke told by a virtual character, how much is he/she laughing about the joke, and how much about the fact that a soulless thing is pretending to tell a joke, employing a dry sysnthetic voice and imprecise mimic? In the latter case, would the user still laugh at the n-th joke? The effect of a behaviour well known on humans is not easy to foresee, when simulated by a virtual human.

First results of a small evaluation study in the Virtual Human related project "Virtual Human with Social Intelligence" (industrial project by ZGDV with SAP AG) have shown that indicators of "pleasure" were significantly high, whereby -perhaps astonishingly-the pleasure was more associated with "relaxation" than with "excitement".

Concerning technically driven effect analysis, further research will be investigated into emotion recognition (see also the article by Encarnacao and Eckes in this special issue) in order to figure out how a user reacts and feels during a conversation with a virtual character.

\section{CONCLUSION}

This article describes the backend of the Virtual Human system, i.e. the application design, the set-up of the two $\mathrm{VH}$ demonstrators (learning scenario 'Life of a star' and 'ZAMB' soccer scenario), underlying methodic-didactic aspects (learning model and ontology) as well as story structuring and the complete authoring process and the integration of the created stories into the $\mathrm{VH}$ run-time system via the narration engine.

Future work will be focused on the facilitation of the authoring process, due to two facts: Costs in the production process and complexity in authoring of interactive media. Additionally, from a Storytelling perspective, further effort will be investigated in the research on the "narrative paradox" addressing the discrepancy between author control (plot based approach with linear media and pre-defined content) and player control (game approach, interactivity, non-linearity).

\section{REFERENCES}

[1] ADDY, 2003. Addy. URL: http://www.addy.de.

[2] A. C. Graesser, P. Chipman, B. C. Haynes and A. Olney. AutoTutor: An Intelligent Tutoring System With Mixed-initiative Dialogue, IEEE Transactions on Education, vol. 48, no. 4, pp. 612- 618, November 2005.

[3] S. Groenewegen and J. Strassner. Virtuelle Charaktere in Lehrsituationen: Ein Konzept zur Nachbildung von realem Unterricht, in 1. Workshop AR VR, im Tagungsband Graphiktag 2004, GI, Chemnitz, 2004.

[4] D. Harel: On Visual Formalisms, Communications of the ACM, vol. 31, no. 5, pp. 514-530, 1988.

[5] R. Hill, J. Gratch, S. Marsella, J. Rickel, W. Swartout and D. Traum. Virtual Humans in the Mission Rehearsal Exercise System., KI special issue on Embodied Conversational Agents, 2003.

[6] I. Iurgel and M. Ziegler. Ask and Answer: An Educational Game where it Pays to Endear You Capricious Virtual Character. IVA 05, International Symposium on Intelligent Virtual Agents, September 12-14, Kos, Greece, 2005.

[7] I. Iurgel. Cyranus-An Authoring Tool for Interactive Edutainment Applications, In: Zhigeng Pan et al. (Ed.). Technologies for E-learning and Digital Entertainment: Edutainment 2006. Berlin, Heidelberg, New York: Springer Verlag, pp. 577-580, LNCS 3942, 2006.

[8] Kircher, Girwidz and Häußler. Physikdidaktik. Eine Einführung in Theorie und Praxis. Vieweg, ISBN 3-528-03100-X, 2000.

[9] Kircher and Schneider. Physikdidaktik in der Praxis, Springer, ISBN 3-540-41937-3, 2002.

[10] S. Klimenko, G. Nielson, L. Nikitina, I. Nikitin and J. Strassner (IMK). Virtual Planetarium: Learning Astronomy in Virtual Reality, in Proceedings of E-learn 2004, Washington, DC, USA, 2004.

[11] M. Mateas and A. Stern. Procedural Authorship: A Case-study of the Interactive Drama Facade, Digital Arts and Culture (DAC), Copenhagen, November 2005.

[12] A. Ortony, G. L. Gnambs and A. Collins. The Cognitive Structure of Emotions, Cambridge University Press, Cambridge, 1988.

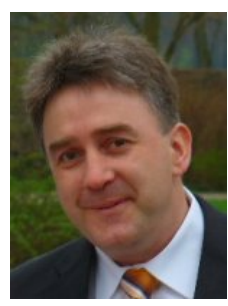

Stefan Göbel received a diploma in computer science at the Technical University of Darmstadt in 1997. From 1997 to 2002 he has been working at the GIS department at the Fraunhofer Institute for Computer Graphics. In 2002 he received a PhD in that domain: "GraphicInteractive Access to Geodata Archives".

Since July 2002, he is the head of the Digital Storytelling department at ZGDV Darmstadt. Here, he is the project leader of numerous RTD activities and projects within the research field of Interactive Digital Storytelling and edutainment applications, e.g. he has coordinated the EU projcect "art-E-fact-Generic platform for the creation of interactive art experience in mixed reality" (FP5) and currently participates in the Integrated Project "INSCAPE-Interactive Storytelling for Creative People" (EU, FP6) and the Co-operative Research Project "U-CREATE-Creative Authoring Tools for Edutainment Applications" (EU, FP6).

Since 2003 he chairs the international conference 'Technologies in Interactive Digital Storytelling and Entertainment' (TIDSE). In 2003 he founded the Forum for Knowledge Media Design (KMD-Forum) as managing director and speaker; in 2006 the KMD-Forum has been settled as working group of the german association for computer science (GI). Since 2005 Dr. Göbel chairs the GameDays event series under the motto 'science meets business'.

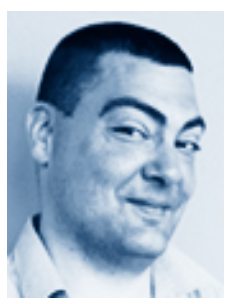

Ido Aharon Iurgel has a master degree in philosophy, social psychology and linguistics from the Ruhr-University of Bochum, and a master in computer sciences from the "Technische Universität Darmstadt". His special interests are concepts for the integration of humanistic sciences and computer science. 1989-1995: Studies on philosophy, social psychology and linguistics at the University of Bochum. Master Thesis on metaphor and emotion.

Since 2002 Ido Aharon Iurgel is a full time scientist (and project manager) at the department. He is responsible for the development of the software platform for narrative applications and of the authoring tool of the department. His work focuses on the development of novel interactive applications that employ virtual characters, and has published several papers on new technologies and novel concepts. He is co-chair of the program of the TIDSE storytelling conference and co-editor of the corresponding LNCS proceedings, and is reviewer of other conferences.

Some of his projects are the EU art-E-fact (Oct. 2002-Febr. 2005, on a mixed virtual humans/real humans discussion group), which he lead scientifically and technically, the ongoing German project Virtual Human, where he leads the contribution of the modules of ZGDV ("Narrative Modules"), as in the Conversational Virtual Humans project, an industrial project in cooperation with SAP. He was responsible for the creation of the Alex talking head for the Heinz-Nixdorf-Museum, and contributed to the IZA information kiosk and to the EMBASSI project, there with expressive talking heads for home assistance. 


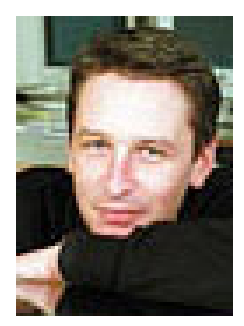

Markus Rössler is one of the founders of rmh new media $\mathrm{GmbH}$. He is managing director and responsible for 3D content in the field of storytelling and virtual reality.

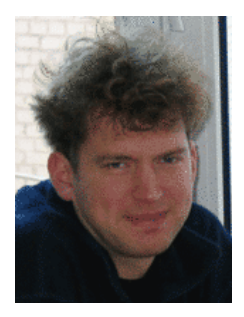

Frank Hülsken is a mathematician with background in pattern recognition. He is working as a research fellow at the Fraunhofer Institute for Intelligent Analysis and Information Systems. His research interests include speech recognition, image processing, 3D reconstruction and animation.

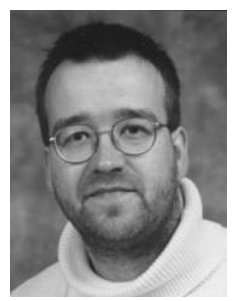

Christian Eckes received the diploma degree in Physics from the University of Dortmund in 1995. From 1996 to 2000 he worked as a research assistant at the Institute for Neural Computation at the Ruhr-University Bochum and, as a visiting scholar, at the University of Southern California (USC), Los Angeles, U.S.A.. In 2001 he joined the Fraunhofer Institute for Intelligent Analysis and Information Systems (IAIS) in the NetMedia group as a research fellow and project manager working in the area of multimedia analysis. His research interests include human-computer interaction, multimedia analysis, pattern recognition and biological inspired computer vision. 